\title{
Nutritional intervention and impact of polyphenol on glycohaemoglobin in type 2 diabetic subjects: systematic review and meta-analysis
}

\author{
S. A. Palma-Duran ${ }^{1}$, A. Vlassopoulos ${ }^{1}$, L. Govan ${ }^{2}$, M. E. J. Lean ${ }^{1}$ and E. Combet ${ }^{1}$ \\ ${ }^{1}$ Human Nutrition, School of Medicine and ${ }^{2}$ Health Economics and Health Technology Assessment Unit, University of \\ Glasgow, G31 2ER, UK
}

Polyphenols have been extensively studied for their antioxidant and anti-inflammatory properties. Recent evidence points toward their antiglycative potential, mediated by modulation of oxidative stress ${ }^{(1-3)}$. So far, the results of human trials investigating the effects of polyphenols on glycation have been inconsistent. Here, we evaluate the published randomized controlled trials (RCTs) of polyphenol supplementation on HbA1c levels in type 2 diabetes mellitus (T2DM) subjects.

A systematic review of all RCTs of polyphenols supplementation in humans with T2DM reporting HbA1c levels before / after supplementation was performed, according to the Preferred Reporting Items for Systematic Review and Meta-Analysis. Twenty-nine RCTs were included. Polyphenol, as extracts or foods, were supplemented ( $28 \mathrm{mg}$ to $1.5 \mathrm{~g}$ ) for a period of 28 days weeks to twelve months. In T2DM subjects $(\mathrm{n}=1426$, mean baseline $\mathrm{HbA} 1 \mathrm{c}=7 \cdot 44 \%, 58 \mathrm{mmol} / \mathrm{mol})$, polyphenol supplementation significantly $(\mathrm{p}<0.001)$ lowered HbA1c $\%$ by $-0.21 \pm 0.04$ units, $(-2.3 \pm 0.04 \mathrm{mmol} / \mathrm{mol})$ compared with the control group (Figure 1$)$.

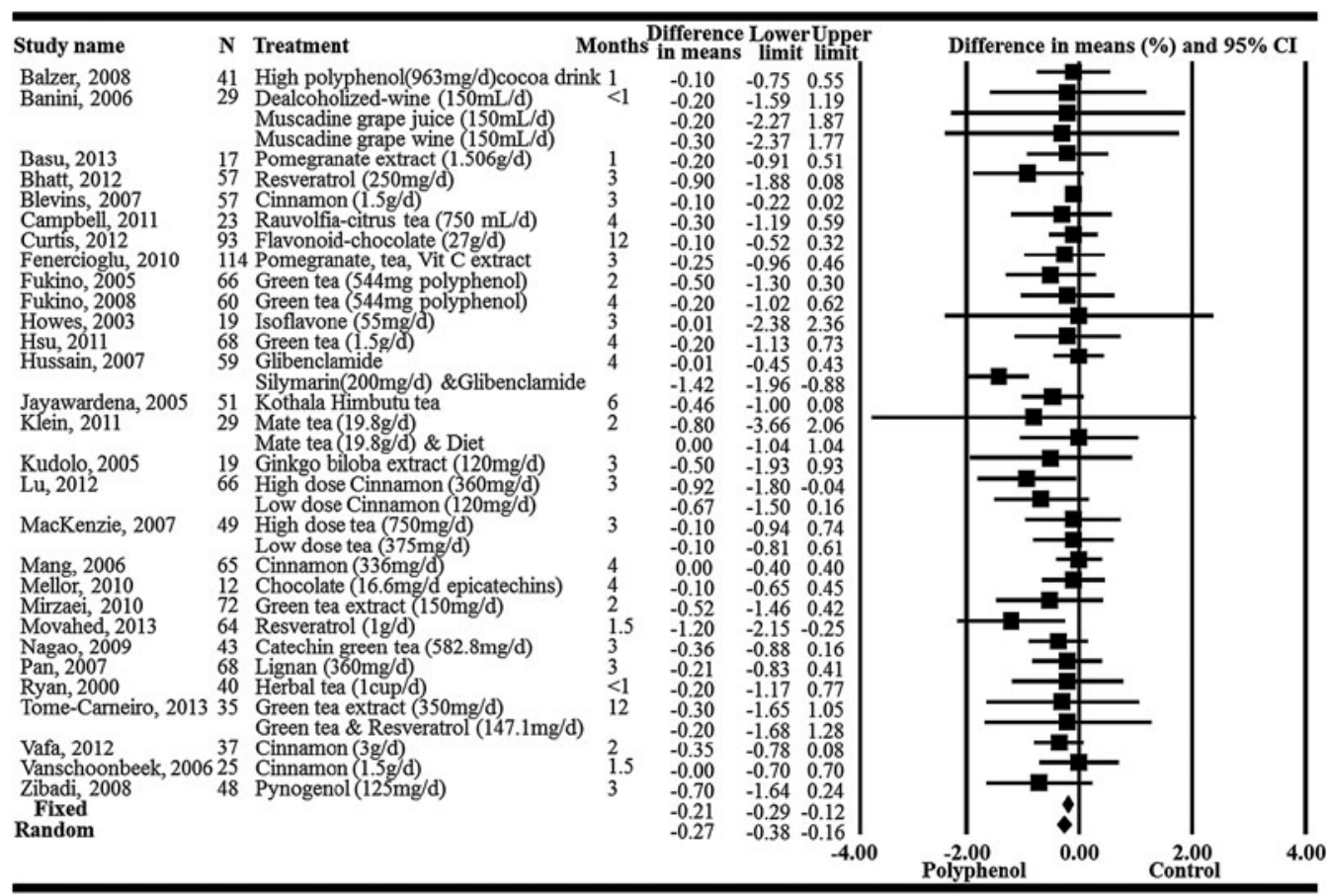

Figure 1. Net change in HbAlc \% associated with polyphenol intervention on T2DM subjects. The overall effect size has been estimated from a fixed-effects model

The doses supplemented are achievable through dietary modification and offer a large range of opportunities for intervention. Nonetheless the trials included had a variety of polyphenol sources, dosage, presentation, duration of the RCTs, heterogeneous group of subjects, and, glucose control level medication, that should be taken into consideration. This meta-analysis highlights the potential of polyphenols as part of T2DM management, with clinical and public health significance that warrants further investigation.

1. Vlassopoulos A, Lean ME, Combet E. (2013) BMC public health 13, Epub 2014/02/07.

2. Sadowska-Bartosz I, Galiniak S, Bartosz G. (2014) Molecules 19, 4880-96.

3. Mandeville JS, Froehlich E, Tajmir-Riahi HA. (2009) J Pharm Biomed Anal 49, 468-74. 